\title{
Frustration effects in magnetic molecules
}

\author{
R. Schmidt ${ }^{\mathrm{a}}$, J. Richter ${ }^{\mathrm{a}}$ and J. Schnack ${ }^{\mathrm{b}}$ \\ a Institut für Theoretische Physik, Universität Magdeburg, P.O. Box 4120, D-39016 Magdeburg, Germany \\ b Universität Osnabrück, Fachbereich Physik, Barbarastr. 7, D-49069 Osnabrück, Germany
}

\begin{abstract}
By means of exact diagonalization we study the ground-state and the low-temperature physics of the Heisenberg antiferromagnet on the cuboctahedron and the icosidodecahedron. Both are frustrated magnetic polytopes and correspond to the arrangement of magnetic atoms in the magnetic molecules $\mathrm{Cu}_{12} \mathrm{La}_{8}$ and $\mathrm{Mo}_{72} \mathrm{Fe}_{30}$. The interplay of strong quantum fluctuations and frustration influences the ground state spin correlations drastically and leads to an interesting magnetization process at low temperatures. Furthermore the frustration yields low-lying nonmagnetic excitations resulting in an extra low-temperature peak in the specific heat.
\end{abstract}

Key words: magnetic molecules, frustration PACS: $75.50 . \mathrm{Xx}, 75.10 . \mathrm{Jm}$

Introduction: The interest in the properties of nanometer-sized magnetic molecules has greatly advanced in recent years [1]. Besides spin rings of various lengths a new route to molecular magnetism is based on highly frustrated magnetic polytopes like the cuboctahedron, Fig. 1, and the icosidodecahedron, Fig. 5. Both are so-called Archimedean solids and magnetic frustration appears due to a triangular arrangement of antiferromagnetic exchange bonds. Recently magnetic molecules have been synthesized having spins located on the vertices of the cuboctahedron $\left(\mathrm{Cu}_{12} \mathrm{La}_{8}\right.$ [2]) and of the icosidodecahedron ( $\left.\mathrm{Mo}_{72} \mathrm{Fe}_{30}[3,4]\right)$. In these frustrated magnetic systems the interplay of topology, interactions and quantum fluctuations may lead to interesting physics at low temperatures $T$ as it is known from quantum spin lattices [5].

The model: We consider a Heisenberg antiferromagnet

$H=J \sum_{(i<j)} \mathbf{s}_{i} \cdot \mathbf{s}_{j}+g \mu_{B} B_{z} S_{z}, \quad J>0$

Email address:

reimar.schmidt@phyisk.uni.magdeburg.de (R. Schmidt). Preprint submitted to Journal of Magnetism and Magnetic Materials to describe the magnetic properties. We set the energy scale by fixing the exchange constant $J=1$. The $\mathbf{s}_{i}$ are the spin operators of the individual magnetic ions, $S_{z}=\sum_{i} s_{i}^{z}$ is the $z$ component of the total spin $\mathbf{S}$ and $B_{z}$ is the magnetic field. We define $h=-g \mu_{B} B_{z}$ and in what follows we use $h$ as parameter of the field. The strength of quantum fluctuations present in the model depends on the spin length $\mathbf{s}_{i}^{2}=s(s+1)$. While $s=$ $1 / 2$ represents the extreme quantum case the system becomes classical in the limit $s \rightarrow \infty$.
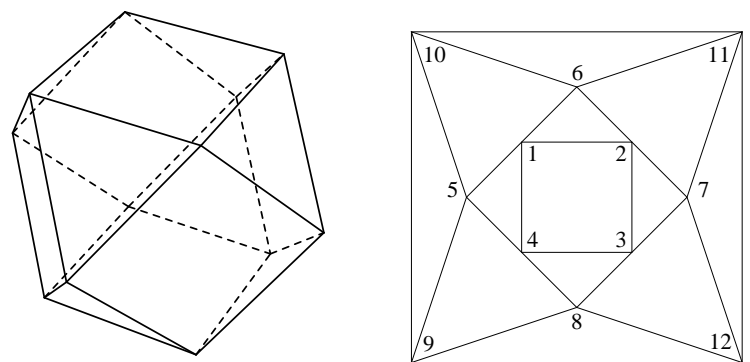

Fig. 1. The cuboctahedron and its planar projection. The spins sitting on the vertices are labeled by numbers. The lines denote exchange couplings $J$.

The Cuboctahedron: The cuboctahedron is shown in 
Fig. 1, left panel. To see the arrangement of exchange bonds more clearly we also show a planar projection in the right panel. For this 12-spin system we are able to find the quantum ground state (GS) for spin quantum numbers $s=1 / 2,1,3 / 2,2,5 / 2,3$ and the full thermodynamics for $s=1 / 2$. In Fig.2 we show the different GS correlators in dependence on the strength of quantum fluctuations measured by $1 / s$. Interestingly, the

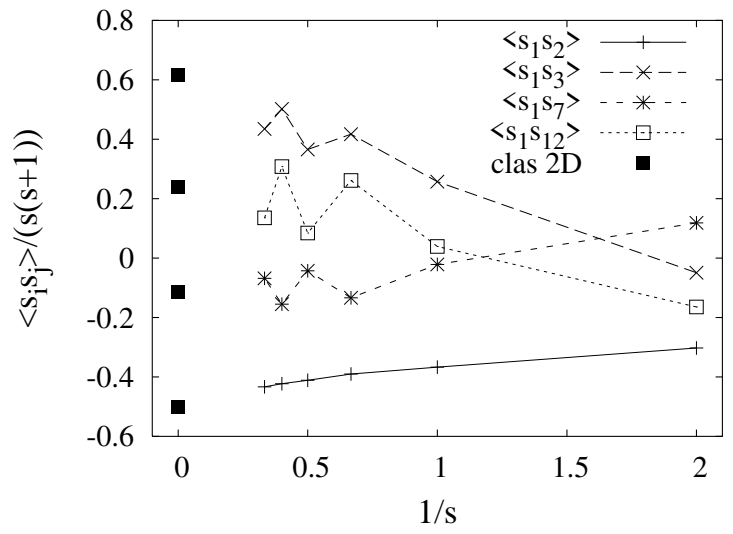

Fig. 2. The four different GS correlators $\left\langle\mathbf{s}_{i} \cdot \mathbf{s}_{j}\right\rangle / s(s+1)$ of the cuboctahedron for $s=1 / 2,1,3 / 2,2,5 / 2,3$ versus $1 / s$. For comparison we present the classical (i.e. $1 / s=0$ ) values obtained by averaging over the degenerate planar (clas) GS's.

extreme quantum case $s=1 / 2$ differs from all other $s$ in the sign of the correlators except for the strong nearest-neighbor correlator which is negative for all $s$. Further we see a qualitative difference between halfinteger and integer spin $s$. This comes from the fact that the three spins on a triangle can be composed to a zero total spin for integer $s$, but cannot for half-integer $s$. The $1 / s$ extrapolation of $\left\langle\mathbf{s}_{i} \cdot \mathbf{s}_{j}\right\rangle / s(s+1)$ approaches the classical value obtained taking into account only planar spin configurations.

Next we consider the magnetization $m=S_{z} /(N s)$ versus field $h$ curve at zero temperature shown in Fig.3. In the classical limit it is a straight line up to saturation field $h_{s} / s=6$. The $m(h)$ curve for quantum model with $s=1 / 2$ differs drastically from the classical straight line. Though for any finite spin system one has step-like $m(h)$ curves there are two special features for the cuboctahedron to be mentioned. The first one is the height of the last step to saturation $m=1$ which is twice as large as the other steps. Again this is a frustration effect and is related to so-called localized magnon states trapped on squares $[1,5,6]$. The second interesting feature is the large width of the plateaus for $m=1 / 3$ and $m=1 / 2$. The existence of plateaus caused by the interplay of frustration and quantum fluctuations has been recently widely discussed for spin

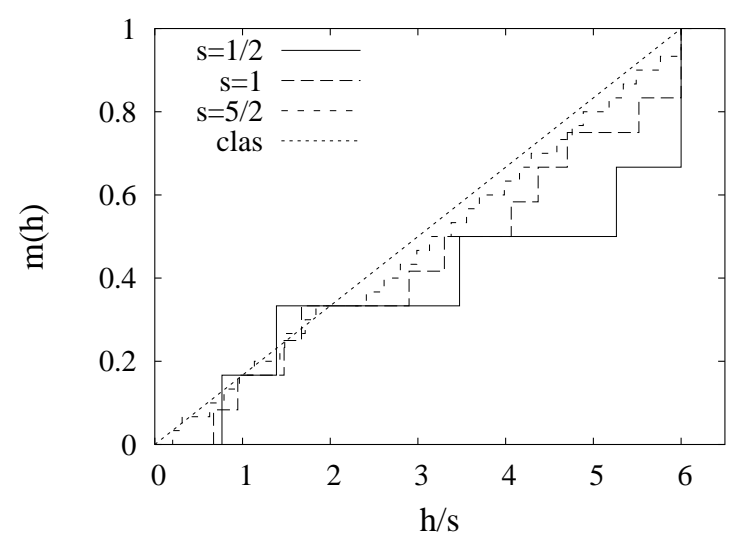

Fig. 3. $T=0$ magnetization $m$ in dependence on $h /(J s)$ for the cuboctahedron with $s=1 / 2,1,5 / 2$ and in the classical case $(s \rightarrow \infty)$.

lattices $[7,5]$. Both features are less pronounced also present for $s=1$ but vanish for larger $s$.

Let us now briefly discuss the specific heat $C$ for the cuboctahedron with $s=1 / 2$ for various strengths of magnetic field $h$ in Fig. 4. The most striking fea-

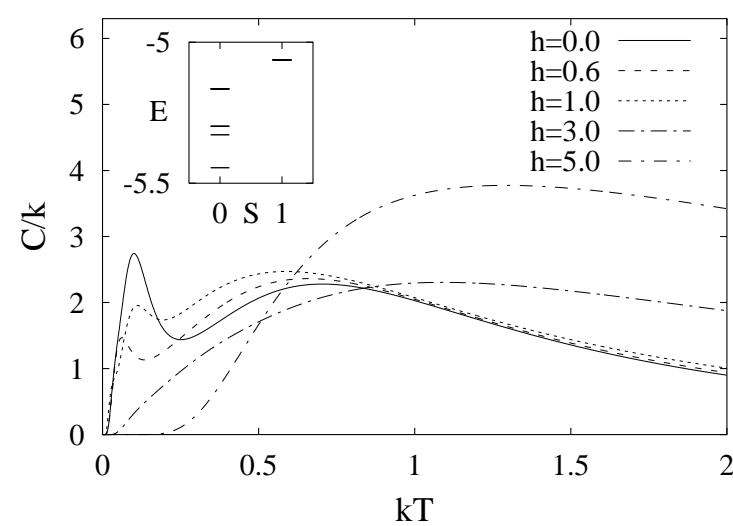

Fig. 4. Specific heat of the cuboctahedron with $s=1 / 2$ versus temperature $T$ for different magnetic fields $h$. The inset shows the lowest energies for $S=0,1$. Note that the third singlet excitation is twofold and the fourth singlet excitation is threefold degenerated.

ture is the low- $T$ peak in $C$ in zero and low fields. The peak disappears for larger fields. We mention, that spin rings do not show such an additional low- $T$ peak in the zero-field specific heat. The existence of such a peak is related to the low-lying spectrum of the system. Contrary to unfrustrated spin rings we find for the cuboctahedron with $s=1 / 2$ at $h=0$ seven low-lying singlet excitations below the first triplet excitation which might be important for the extra peak (see the inset in Fig. 4). 
The icosidodecahedron: Now we consider the icosidodecahedron, Fig. 5, left panel. To see the arrangement of exchange bonds more clearly we also show a planar projection in the right panel. For this
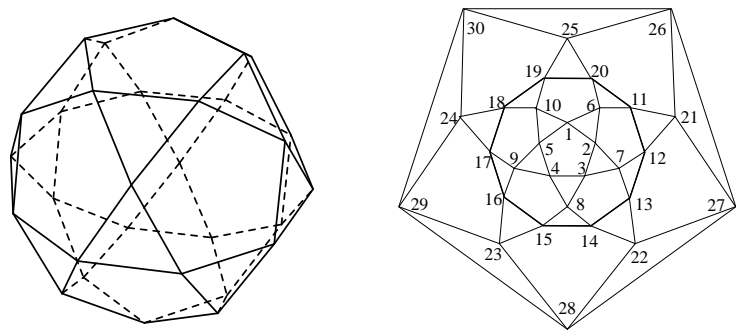

Fig. 5. The icosidodecahedron and its planar projection. The spins sitting on the vertices are labeled by numbers. The lines denote exchange couplings $J$.

30-spin system we are able to find the quantum GS at arbitrary magnetic field $h$ for spin quantum numbers $s=1 / 2$ only. For larger spin $s$ we are able to calculate the GS for large fields only. The GS correlators for $s=1 / 2$ at zero field are $\left\langle\mathbf{s}_{1} \mathbf{s}_{2}\right\rangle=-0.22057$, $\left\langle\mathbf{s}_{1} \mathbf{s}_{3}\right\rangle=-0.00026,\left\langle\mathbf{s}_{1} \mathbf{s}_{7}\right\rangle=0.05915,\left\langle\mathbf{s}_{1} \mathbf{s}_{8}\right\rangle=$ $0.00011,\left\langle\mathbf{s}_{1} \mathbf{s}_{13}\right\rangle=-0.03609,\left\langle\mathbf{s}_{1} \mathbf{s}_{14}\right\rangle=-0.00184$, $\left\langle\mathbf{s}_{1} \mathbf{s}_{22}\right\rangle=0.02097,\left\langle\mathbf{s}_{1} \mathbf{s}_{28}\right\rangle=-0.03592$. Obviously, all correlations except the nearest-neighbor one are weak as a result of frustration and quantum fluctuations.

In Fig. 6 we show the magnetization $m=S_{z} /(N s)$ versus field $h$ curve at $T=0$. Again for $s \rightarrow \infty$ it is a

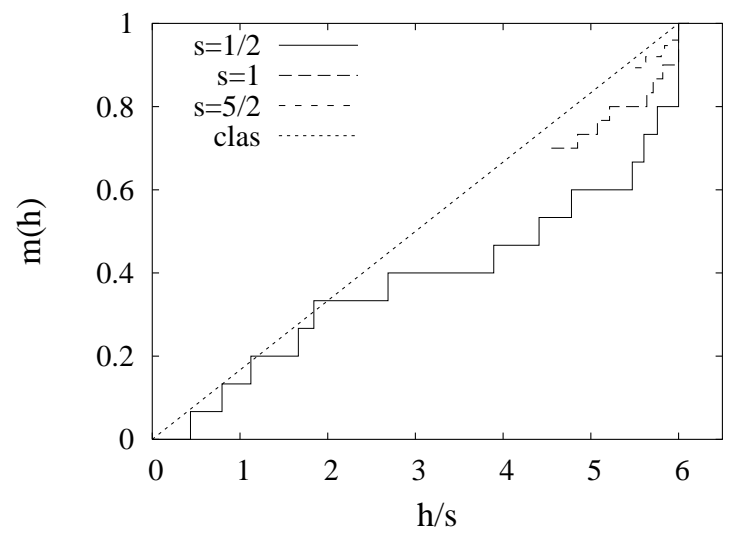

Fig. 6. $T=0$ magnetization $m(h)$ in dependence on $h /(J s)$ for the icosidodecahedron with $s=1 / 2,1,5 / 2$ and in the classical case $(s \rightarrow \infty)$.

straight line up to saturation field, but for $s=1 / 2$ it is completely different. Like for the cuboctahedron the $m(h)$ curve for $s=1 / 2$ shows an extra high jump to saturation due to localized magnon states and plateaus of large width at $m=3 / 5,2 / 5,1 / 3$. For $s=1$ and $s=5 / 2$ we show the high-field part of $m(h)$. The jump and the plateaus are also seen for $s=1$, but less pronounced. For $s=5 / 2$ the $m(h)$ curve approaches the classical line.

Though we cannot calculate thermodynamic quantities we are able to present the low-energy spectrum, relevant for the low- $T$ physics, for the icosidodecahron with $s=1 / 2$ (Fig. 7 ). The lowest states in each sec-

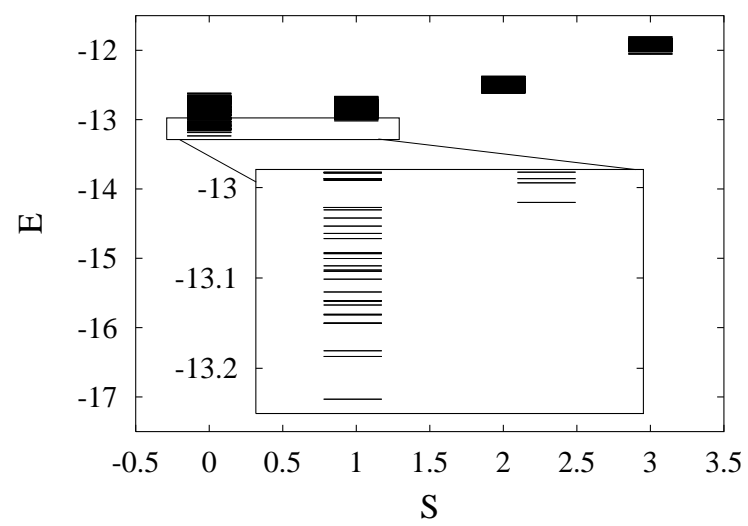

Fig. 7. Low-lying levels versus quantum number of total spin $S$ for the icosidodecahedron with $s=1 / 2$. The inset shows the singlets $(S=0)$ below the first triplet $(S=1)$ on an enlarged scale.

tor of the total spin $S$ are well described by rotational bands, i.e. we have approximately $E_{\min }(s) \propto S(S+1)$ [1]. As shown in the inset there are more than 20 singlets $(S=0)$ below the first triplet $(S=1)$ excitation. This large number of low-lying singlets is due to frustration and was also found for the cuboctahedron with $s=1 / 2$. Note that unfrustrated rings do not have singlet excitations below the first triplet. Hence we may expect that for the icosidodecahedron also an extra low- $T$ peak in $C$ appears. We mention, however, that for the low- $T$ behavior of the susceptibility the singlets are irrelevant.

Acknowldegement: We are indebted to J. Schulenburg for assistance in numerical calculations.

\section{References}

[1] J. Schnack, Molecular Magnetism, in Quantum Magnetism, Lecture Notes in Physics 645, U. Schollwöck, J. Richter, D.J.J. Farnell, R.F. Bishop, Eds. (Springer-Verlag, Berlin, 2004), p. 155 - 194

[2] A.J. Blake, R.O. Gould, C.M Grant, P.E.Y Milne, S. Parsons, R.E.P. Winpenny, J.Chem. Soc.- Dalton Trans. 4, 485 (1997)

[3] A. Müller, S. Sarkar, S.Q.N. Shah, H. Bögge, M. Schmidtmann, Sh. Sarkar, P. Kögerler, B. Hauptfleisch, A.X. Trautwein, V. Schünemann, Angew. Chem. Int. Ed. Engl., 38, 3238 (1999) 
[4] A. Müller, M. Luban, C. Schröder, R. Modler, P. Kögerler, M. Axenovich, J. Schnack, P.C. Canfield, S. Bud'ko, N. Harrison, Chem. Phys. Chem., 2, 517 (2001)

[5] J. Richter, J. Schulenburg, A. Honecker, Quantum magnetism in two dimensions: From semiclassical Néel order to magnetic disorder; in Quantum Magnetism, Lecture Notes in Physics 645, U. Schollwöck, J. Richter, D.J.J. Farnell, R.F. Bishop, Eds. (Springer-Verlag, Berlin, 2004), p. 85 - 183 [http://www.tu-bs.de/ 〜honecker/papers/2dqm.ps.gz].

[6] J. Schulenburg, A. Honecker, J. Schnack, J. Richter, H.-J. Schmidt, Phys. Rev. Lett. 88, 167207 (2002)

[7] A. Honecker, J. Schulenburg and J. Richter, J. Phys.: Condens. Matter 16, S749 (2004) 